\title{
NOTE ON THE ORTHOTOMIC CURTE OF
} A SYSTEM OF LINES IN A PLANE.

\author{
By Prof, Cayley.
}

Considering in plano a singly infinite system of lines, then to each point of the plane there corresponds a line (not in general a unique line), and we can therefore express in terms of the coordinates $(x, y)$ of the point the cosine-inclinations $\alpha, \beta$ of the line to the axes. The differential equation of the orthotomic curve is then $\alpha d x+\beta d y=0$, and it is a well-known and easily demonstrable theorem that $\alpha d x+\beta d y$ is a complete differential, say it is $=d V$; the integral equation of the orthotomic curve is therefore $V=\int(\alpha d x+\beta d y)$, = const., and we see further that $V$ is a solution of the partial differential equation $\left(\frac{d V}{d x}\right)^{2}+\left(\frac{d V}{d y}\right)^{2}=1$.

If the lines are the normals of the ellipse $\frac{X^{2}}{a}+\frac{Y^{2}}{b}=1$, then, writing the equation of the normal at the point $X, Y$ in the form

$$
\frac{a}{X}(x-X)=\frac{b}{Y}(y-Y),=\lambda \text { suppose, }
$$

we have

$$
X=\frac{a x}{a+\lambda}, Y=\frac{b y}{b+\lambda} ;
$$

and therefore $\frac{a x^{2}}{(a+\lambda)^{2}}+\frac{b y^{2}}{(b+\lambda)^{2}}-1=0$,

which last equation determines $\lambda$ as a function of $x, y$. We have $\alpha, \beta$ proportional to $\frac{X}{a}, \frac{Y}{b}$; or say we have $\alpha=M \frac{x}{a+\lambda}$, $\beta=M_{b} \frac{y}{b+\lambda}$, whence $\frac{1}{M^{2}}=\frac{x^{2}}{(a+\lambda)^{2}}+\frac{y^{2}}{(b+\lambda)^{3}} ;$ or, writing for couvenience

$$
\frac{x^{2}}{(a+\lambda)^{2}}+\frac{y^{2}}{(b+\lambda)^{2}}-\frac{k^{2}}{\lambda^{2}}=0,
$$

(viz., this equation defines $k$ as a function of $x, y$ and $\lambda$, that is of $x$ and $y$ ), we have

and we ought therefore to have

$$
\alpha=\frac{\lambda x}{k(a+\lambda)}, \quad \beta=\frac{\lambda y}{k(b+\lambda)} ;
$$

$$
\frac{\lambda}{k}\left(\frac{x d x}{a+\lambda}+\frac{y d y}{b+\lambda}\right)
$$

a complete differential, $=d V$. 
This is easily verified, for from the assumed value

we deduce

$$
k=\lambda\left(\frac{x^{2}}{a+\lambda}+\frac{y^{2}}{b+\lambda}-1\right)
$$

$$
\begin{aligned}
d l c & =2 \lambda\left(\frac{x d x}{a+\lambda}+\frac{y d y}{b+\lambda}\right)+d \lambda\left(\frac{a x^{2}}{(a+\lambda)^{2}}+\frac{b y^{2}}{(b+\lambda)^{3}}-1\right), \\
& =2 \lambda\left(\frac{x d x}{a+\lambda}+\frac{y d y}{b+\lambda}\right)
\end{aligned}
$$

and we have therefore

$$
d V=\frac{\lambda}{k} \frac{d k}{2 \lambda},=\frac{1}{2} \frac{d k}{k}
$$

where $k$ denotes a function of $(x, y)$ defined as above; hence the equation $V=$ const. gives $k=$ const, or the equation of the orthotomic curve is given by the system of equations

$$
\begin{aligned}
& \frac{a x^{2}}{(a+\lambda)^{3}}+\frac{b y^{2}}{(b+\lambda)^{2}}-1=0, \\
& \frac{x^{2}}{(a+\lambda)^{2}}+\frac{y^{2}}{(b+\lambda)^{2}}-\frac{k^{2}}{\lambda^{3}}=0,
\end{aligned}
$$

where $k$ is a constant; these equations (eliminating $\lambda$ ) give in fact the equation of the parallel curve of the ellipse, and $k$ denotes the normal distance of a point on the curve from the ellipse. I recall that the first equation may be replaced by

$$
\frac{x^{2}}{a+\lambda}+\frac{y^{2}}{b+\lambda}-\frac{k}{\lambda}-1=0
$$

and since the derived equation hereof in regard to $\lambda$ is the second equation, we have the equation of the parallel curve in the known form

Disct. $\left\{(\lambda+k)(\lambda+a)(\lambda+b)-(b+\lambda) x^{2}-(a+\lambda) y^{\prime \prime}\right\}=0$.

I notice further that considering $k$ a function of $x, y$ as above, we have

$$
\begin{aligned}
\left(\frac{d V}{d x}\right)^{2}+\left(\frac{d V}{d y}\right)^{2}=\frac{1}{4 k^{2}}\left\{\left(\frac{d k}{d x}\right)^{2}+\left(\frac{d k}{d y}\right)^{2}\right\}, & \\
= & \frac{\lambda^{2}}{k^{2}}\left\{\frac{x^{8}}{(a+\lambda)^{2}}+\frac{y^{2}}{(b+\lambda)^{2}}\right\}, \text { that is }\left(\frac{d V}{d x}\right)^{2}+\left(\frac{d V}{d y}\right)^{2}=1,
\end{aligned}
$$

as it should be. 